\title{
Modelling and Controlling the Compliance of a Robotic Hand with Soft Finger-pads
}

\author{
L. Biagiotti ${ }^{1}$, P. Tiezzi ${ }^{2}$, G. Vassura ${ }^{2}$, and C. Melchiorri ${ }^{1}$ \\ 1 DEIS, University of Bologna \\ Via Risorgimento 2, \\ 40136 Bologna, Italy \\ \{lbiagiotti, cmelchiorri\}@deis.unibo.it \\ 2 DIEM, University of Bologna \\ Via Risorgimento 2, \\ 40136 Bologna, Italy \\ \{paolo.tiezzi, gabriele.vassura\}@mail.ing.unibo.it
}

\begin{abstract}
Compliant pads greatly contribute to increase the robustness and the stability of grasps of robot hands, because of their conformability to the objects' surfaces, the capability to damp dynamic effects and to dissipate repetitive strains, the enlarged contact areas they allow. On the other hand, besides the difficulty to obtain a precise model of soft pads, they appears not suitable to achieve stiff and accurate grasps in those tasks that require high stiffness and precision.

In this paper, the normal and tangential stiffnesses of soft materials have been experimentally investigated in order to demonstrate their suitability with the development of compliant pads for robotic hands. Since these stiffnesses strongly depends on the applied load, a control approach, exploiting such relation, is proposed in order to "arbitrarily" change the overall stiffness of the hand. In this sense, the perspective of this control strategy, differently from the traditional one that "makes more compliant a stiff system", is to use the internal forces of the grasp to "make stiffer a compliant system".
\end{abstract}

\section{Introduction}

In recent years, the trend in robot hand design has been oriented towards devices inspired to biological models and, in particular, to human hand. The traditional criteria, which led to the development of the early prototypes of dexterous robotic hands [1-3], clearly tied to the same design guidelines used for industrial robots, have been substituted in recent applications by more suitable design approaches. In this new perspective, in lieu of rigid kinematic chains more compliant mechanical structures have been adopted. Moreover, the importance of thick compliant layers of visco-elastic material has been widely recognized in order to enhance the grasp and manipulation capabilities of dexterous hands and some robotic fingers/hands equipped with soft pads have been recently developed [4,5].

Due to their conformability, compliant materials allow to adapt on objects with uneven surfaces and to distribute contact forces, avoiding damages to the grasped objects. Soft pads allow to better sustain and damp dynamic effects, like shocks and vibrations, and to better dissipate repetitive strains, that are induced during 
manipulation [6,7]. Compliant materials have large coefficients of friction, making it possible to use lower gripping forces with indirect advantages about sizing of actuators [8]. Furthermore compliant pads allow enlarged contact areas that involve a kinematical coupling between the object and the finger, so that a lower number of contacts, with respect to hard fingers, can guarantee to restrain an object [9]. In general, soft pads increase the grasp robustness and stability.

On the other hand, there are some drawbacks that must be considered in developing robotic fingers with soft pads: the difficulty to model their behavior characterized by visco-elastic phenomena, nonlinearities, the difficulty to obtain suitable stiff and accurate grasps in tasks that require high level of stiffness and precision, and so on.

Some important suggestions, toward the development of robotic fingers with soft pads, can be obtained from the analysis of the human finger behavior. As observed in previous works $[10,11]$ human fingers exhibit a strong nonlinear mechanical behavior. Two of the main causes are the presence of the bone and the nail that constrain the deformation of pad tissues. The stiffness of the human finger increases nonlinearly with increasing flattening of the pad (even if it is small under little displacements it grows quickly), simultaneously the contact area changes with grasping force and this change influences the overall stiffness values of the finger. Moreover the stiffness changes with the contact angle between the fingertip and the object due to the presence of the nail [12].

Robotic fingers, that are inspired by the biological model, are characterized by an internal rigid structure covered by a soft layer and, possibly, by an external epidermal layer. Most of the previous models and experimental researches $[6,13]$ consider an hemispherical fingertip completely made of homogeneous material; even though the contact model that better describes the situation is the one reported in Fig. 1 (see [14]), in which the body (b) is in contact with the compliant layer (a) placed on a substrate (c). The bodies (b) and (c) are rigid while the layer (a) behaves elastically. As the flattening of soft pad increases the rigid substrate becomes more and more dominant and it is really important to take into account its influence on the soft layer deformation.

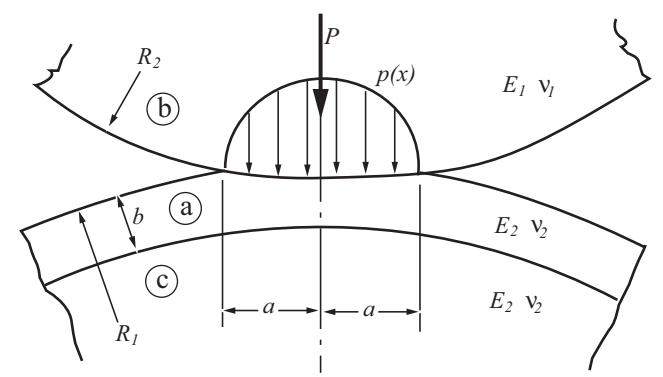

Fig. 1. Contact model of a robotic finger with an inner rigid "bone" (c) covered by a soft pad (a) during grasping of an object (b). 
The main aspects, that affect the contact mechanics of the presented model, are:

- the material (especially its softness);

- the thickness of the soft layer;

- the radius of curvature of the pad.

In [6], the authors explicitly point out that in some industrial applications, soft fingers and soft jaws are unacceptable and, in general, the presence of soft tissues is regarded as a reason of poor precision for the overall system. Aim of the second part of this work is to demonstrate that a robot hand, which, in principle, is considered compliant (due to soft pads, or, more generally, to elastic elements included in the fingers' structure), can be made "arbitrarily" stiff. For this purpose, the non-linear behavior of the pads plays a key-role.

As a matter of fact, a robotic end-effector (and in particular a multifingered robot hand) is conceived to grasp and use generic objects and tools, e.g. a screwdriver or the handle of a door. Therefore the total compliance of the device/object, which is one of the most important quantities for evaluating the grasp, must be considered. Such a kind of investigation has been already performed by Cutkosky and Kao [15], but their analysis is based on devices characterized by very low level of structural compliance. When very compliant elements (pads) are included in the mechanical structure, the overall compliance is still obtained by simply summing up the contribution of each element (control algorithms, actuators, motion transmissions, hand skeleton, pads, and so on...) but it is not possible to arbitrarily modify such a compliance by simply changing the stiffness of the control strategies. The total compliance of the hand will be bounded from below by the compliance (usually very large) of the finger-pads and a procedure aiming "to make rigid a compliant device" [16] is needed.

The control approaches adopted for "traditional" robot hands (which in many cases neglect the structural compliance of the device, supposed very small) should be revised in order to explicitly take into account this issue. The control strategy, proposed in this work, is based on two main steps:

- The stiffness of the pads (usually treated as a constant) is modulated by exploiting the internal force of the grasp;

- The desired compliance of the hand is imposed by acting on the control gains.

In this way, it is possible to augment the stiffness of the soft pads and carry out very rigid grasps.

\section{Modelling of soft pad stiffness: experimental tests}

\subsection{The fingertip specimens}

In order to investigate the stiffness of soft pads, under normal and tangential loads, specimens of simplified purposely-shaped fingertips have been tested. The simplified fingertips (see Fig. 2) have a hemispherical geometry and a constant thickness of the soft layer bounded to the rigid inner structure. The experimental activity allowed to 
investigate the influence of the design parameters (the thickness of soft pads) and material (its softness) comparing two different elastic silicon rubbers:

A) soft silicon rubber (hardness 18 Shore A);

B) very soft silicon rubber (hardness 20 Shore 00).

For each material three different thicknesses of the soft pad have been adopted $(1,5 \mathrm{~mm} ; 3 \mathrm{~mm} ; 6 \mathrm{~mm})$ with the same external radius of curvature $(10 \mathrm{~mm})$.

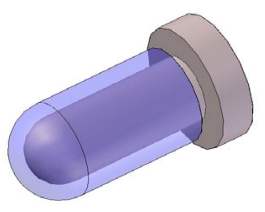

(a)

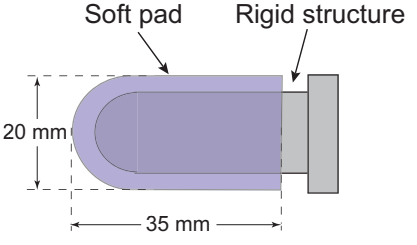

(b)

Fig. 2. Structure of the tested soft fingertip.

\subsection{Experimental equipment}

Two different tests have been performed in order to investigate the contact mechanics under normal and tangential loads.

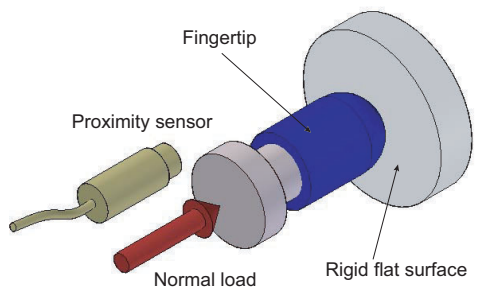

(a)

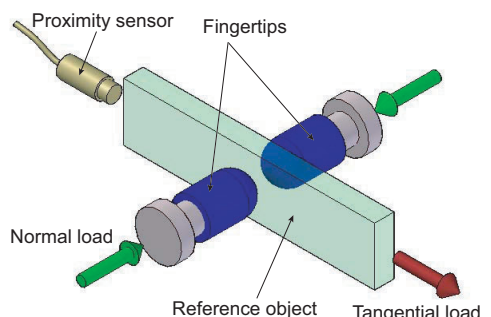

(b)

Fig. 3. Illustration of the experimental tests.

With reference to Fig. 3.a, the fingertip is pressed against a flat rigid surface and the flattening, which occurs under the normal load, is monitored. 
With reference to Fig. 3.b, two fingertips are symmetrically applied on the opposite faces of a reference object in order to investigate the contact mechanics under normal and tangential loads. Besides the evaluation of contact robustness with respect to linear sliding due to the overcoming of friction limits, the analysis of object displacement can provide useful measure of the normal and tangential stiffness of contact.

The test equipment (see Fig. 4) is composed by two counteracting four-bar linkages, with low friction joints, that guarantees the symmetry of both displacements and loads.

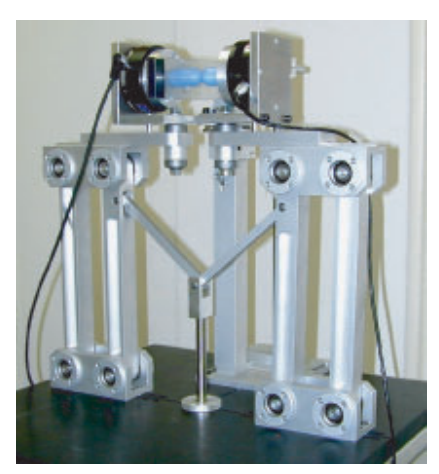

(a)

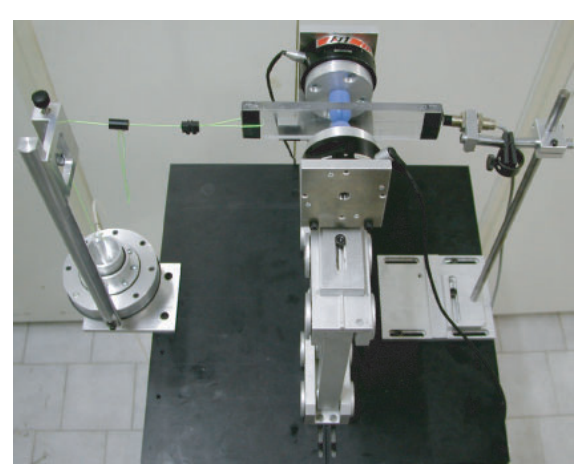

(b)

Fig. 4. The purposely designed equipment adopted to perform the experimental test. A comprehensive view of the mechanism (a). The setup adopted for the tangential tests (b).

Between each fingertip and the relative holding linkage a multi-component forcetorque sensor is placed that allows complete monitoring of the applied forces and torques. A system of contact-less proximity sensors is adopted in order to continuously monitor the displacements of both the fingertip and the reference object. A rolling-diaphragm pneumatic actuator applies disturbance loads on the reference object, while on the fingertips the loads, normal to contact surface, are applied to each linkage by weights activated by a second pneumatic cylinder. The system guarantees a correct approach to the contact surface and a good control of the applied loads with acceptable repeatability.

Advantages of such testing concept are the capability to perform tests both on surface properties and on static and dynamic behavior of the pads, the absence of disturbing effects, the complete reproduction of operating conditions, the relatively low complexity of test equipment [17]. 


\subsection{Experimental results}

Normal Tests The experimental results are reported in the following figures. Fig. 5.a shows the relation between the applied normal force and the flattening of the soft pad; Fig. 5.b shows how the stiffness varies with the flattening of pad. In the same graphics the behavior of soft pads is compared with that of the frame, that represents a reference of the behavior of a mechanical rigid structure.

It can be observed that all soft pads behave nonlinearly. The pads formed by the softer rubber are more influenced, respect to the harder pads, by the inner rigid core. The presence of the rigid "bone" involves a significant increase in the stiffness of the pad as the load becomes more intense (see Fig. 6). This implies that, when the task requires, it is possible to obtain a larger stiffness by increasing appropriately the grasp forces.

Tangential Tests Fig. 7 shows the experimental results for the silicon rubber (B) whit a thickness of the pad equal to $3 \mathrm{~mm}$. Fig. 7.a shows the relation between the tangential load and the tangential displacement, considering different normal forces. Fig. 7.b shows the relation between tangential stiffness $\partial \mathrm{T} / \partial \mathrm{x}$ and normal load $\mathrm{N}$.

From the experimental results it comes out that, maintaining constant the normal load, before slippage there is a linear dependence between the displacement and the load acting tangentially. A constant stiffness, that correlates the tangential force and the displacement along the same direction, can be found. Nevertheless, as the normal load changes, a dependence between the tangential stiffness and the normal load appears (see Fig. 7.b). When slippage happens the load suddenly decreases and stick-slip phenomena occurs.

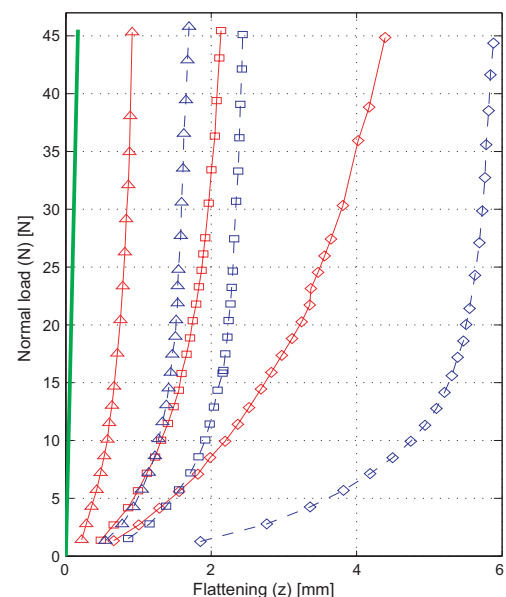

(a)

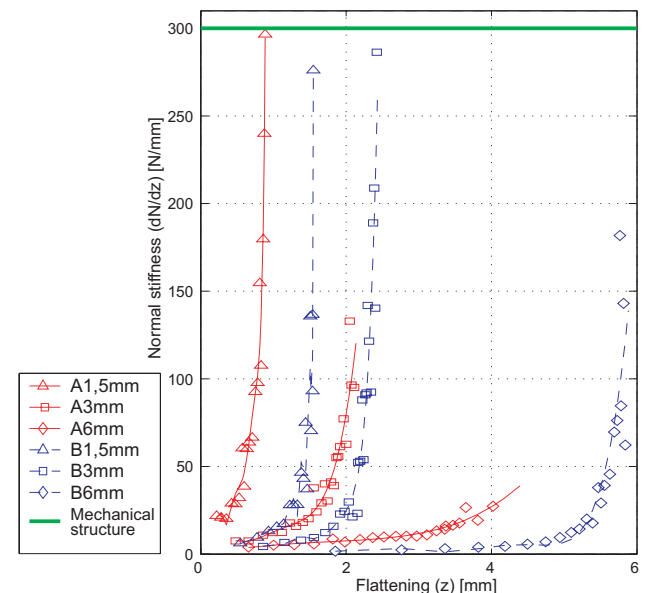

(b)

Fig. 5. Experimental results: flattening vs. normal load (a) and relative stiffness (b). 
Fig. 8.a shows the comparison between two different thicknesses of the rubber (B) at different normal loads. Fig. 8.b shows the comparison between the two material at different normal loads maintaining constant the thickness $(3 \mathrm{~mm})$. Both softness and thickness of the covering material affect the tangential stiffness of the artificial finger. Decreasing the thickness of the soft layer, the overall behavior of the fingertip becomes more rigid and the tangential stiffness increases. Nevertheless, because of the contact area decreases, the maximum value of the tangential load, that can be sustained, decreases.

Stiffness model In the following discussion the rubber (B) with a thickness of $3 \mathrm{~mm}$ is adopted as reference. As discussed above, both normal and tangential stiffness depend on the normal load acting on fingertip and both increase quickly with the normal load. A number of models have been proposed to represent the stiffness of soft layers, with different purposes and level of details [10], [18]. In our case, a model of the normal and the tangential stiffness has been obtained by fitting the experimental results through the least square method using the simple biparametric expression:

$$
k=a \cdot e^{b \cdot N}
$$

where $a$ and $b$ are parameters. The normal stiffness is

$$
k_{n}=6.963 \cdot e^{0.097 \cdot N}
$$

In Fig. 6 the calculated values are compared with the experimental ones. The tangential stiffness is

$$
k_{t}=0.287 \cdot e^{0.063 \cdot N}
$$

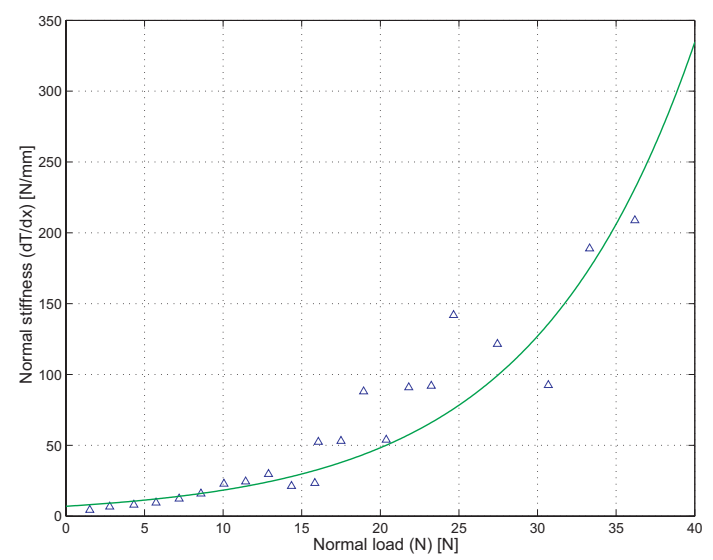

Fig. 6. Comparison between experimental and calculated results for the rubber B with a thickness of the layer equal to $3 \mathrm{~mm}$. 




(a)



(b)

Fig. 7. Experimental results for the rubber B with a thickness of $3 \mathrm{~mm}$ : tangential load vs. tangential displacement (a) and comparison between experimental and calculated results for tangential stiffness vs. normal load (b).

In Fig. 7.b the experimental values and those calculated are compared.

These considerations point out that, by modulating the normal load, a change in both normal and tangential stiffness can be obtained.

\section{Stiffness control of the fingertips: the basic idea}

One of the most popular control modalities for robotic hands is stiffness control and, more generally, impedance control. Aim of this class of controllers is to change the relation between forces and velocities/positions, which characterizes a robotic device, in order to achieve a prescribed behavior. In particular, stiffness control acts on the static relation force/positon according to the task to be performed.

As well known [15], the overall stiffness and, more generally, the complete dynamic behavior of a robotic system (in our case a finger) result from the contributions of:

- the actuation system and the control modalities;

- the motion transmission and the mechanical structure;

- the interface between the tip and the environment;

- the change in contact geometry as well as fingers' configuration.

Considering the overall model of a robotic finger, as reported in Fig. 9, in the simple case of a single degree of freedom, these "elastic" elements (with the only exception of the geometric changes) work in series. Therefore

$$
K_{\text {tot }}^{-1}=K_{c}^{-1}+K_{s}^{\prime-1}+K_{s}^{\prime \prime-1}+\ldots+K_{p}^{-1}
$$




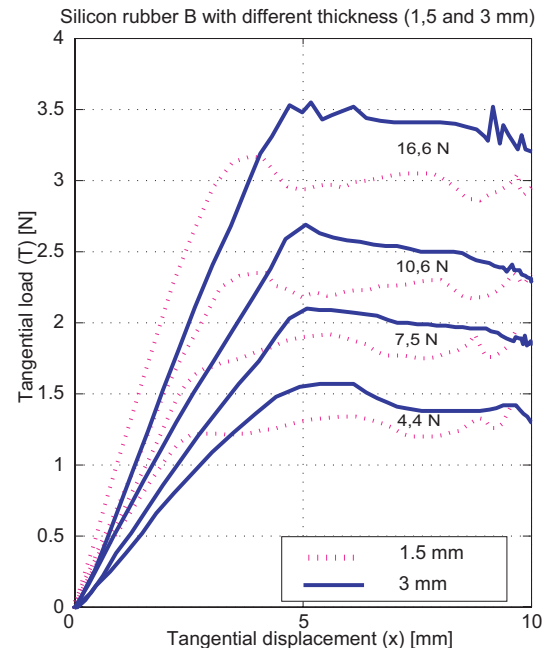

(a)



(b)

Fig. 8. Comparison of the behavior under tangential load between different thickness of the same material (a), and between different materials with the same thickness (b).

where $K_{\text {tot }}$ is the total stiffness of the system, $K_{c}$ the virtual stiffness of the controller, $K_{s}^{\prime}$ and $K_{s}^{\prime \prime}$ represent the elastic elements of the mechanical structure (e.g. tendons, joints, etc.) and $K_{p}$ is the stiffness of the soft pad. For the sake of simplicity, in eq. (4) the transformations from joint space to cartesian space are not reported.

In general, stiffness control approach acts by reducing the overall stiffness of the system, which otherwise will be quite rigid. Therefore, if a robotic hand is considered, this kind of controller assumes that the fingers are structurally very stiff $\left(K_{s}^{\prime}, K_{s}^{\prime \prime}\right.$ very high). Such an hypothesis does not fit with the adoption of a soft layer of viscoelastic materials at the interface between fingertips and objects. As a matter of fact (see eq. 4) a soft element such as a fingertip pad, whose compliance $\left(\Lambda_{p}=K_{p}^{-1}\right)$ is generally very high, produces a lower bound on the overall compliance of the

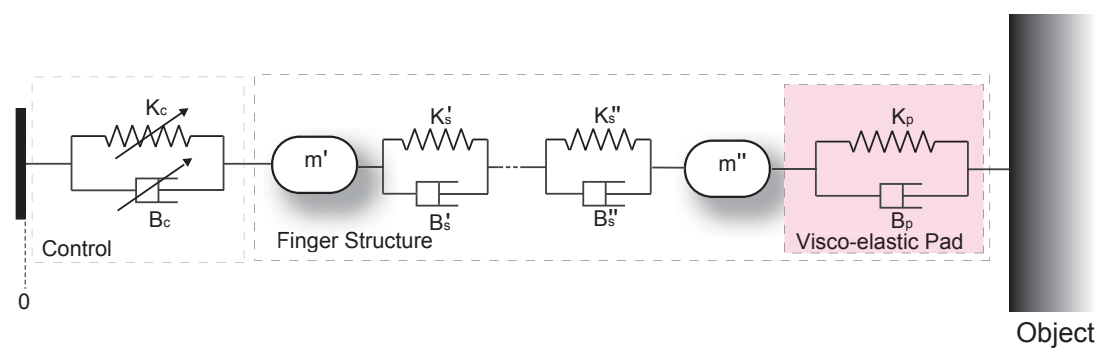

Fig. 9. Schematic model of a 1-dof robotic finger. 




Fig. 10. Schematic model of a 1-dof robotic finger, with the only contributions of control and soft interface.

system, being all the elements in eq. 13 positive.

The perspective of the controller is, therefore, different from the traditional one: we want "to make stiffer a compliant system" and not "to make more compliant a stiff system", as usual.

\subsection{An introductory example}

Since in this analysis we are mostly interested in the effects of the soft pads, a simplified model (depicted in Fig. 10) is considered, where the compliance of the structure is neglected and the only effects taken into account are those due to the control and the pads themselves. In addition, we have considered the effects of the friction $\left(f_{f}\right)$, which dramatically affects robotic end-effectors (because of the high reduction ratio of the transmission system, the use of tendons, and so on) and plays a key role on the definition of the overall stiffness.

The dynamic model of the robotic finger is

$$
m \ddot{x}=K_{c}\left(x_{0}-x\right)+K_{p}\left(x_{w}-x\right)+B_{c}\left(\dot{x_{0}}-\dot{x}\right)+B_{p}\left(\dot{x_{w}}-\dot{x}\right)+f_{f}
$$

where $K_{c}\left(x_{0}-x\right)$ and $B_{c}\left(\dot{x_{0}}-\dot{x}\right)$ are the elastic and the damping terms due to the control (with $x_{0}$ reference position), $K_{p}\left(x_{w}-x\right)$ and $B_{p}\left(\dot{x_{w}}-\dot{x}\right)$ represent the visco-elastic behavior of the finger pad, $m$ is the mass of the finger, $x_{w}$ the position of the grasped object, supposed rigid. Two different situations are considered:

1. only positions sensors are used for the control (while proximity sensors or force sensors can be profitably used during the planning phase);

2. force sensors are available for control purposes. 
Stiffness control with position sensors The stability of system can be easily inferred ${ }^{1}$ from (5), but from the Lasalle's principle it follows that an invariant set for the system is

$$
x=\frac{K_{c} x_{0}+K_{p} x_{w}}{K_{c}+K_{p}}+\frac{f_{s}}{K_{c}+K_{p}}
$$

where it is possible to recognize two terms: the former $\left(x^{*}=\frac{K_{c} x_{0}+K_{p} x_{w}}{K_{c}+K_{p}}\right)$ related to the stiffness of the overall system and the latter $\left(\Delta x^{*}=\frac{f_{s}}{K_{c}+K_{p}}\right)$ depending on the stiction $\left(f_{f}=f_{s}\right.$ for $\left.\dot{x}=0\right)$. This reflects the fact that there is not a unique equilibrium point but in steady-state the position of the finger is

$$
x \in x^{*} \pm \Delta x_{\max }^{*}
$$

In particular the force applied to the object is

$$
f_{c}=K_{t i p} d+\frac{K_{p}}{K_{c}+K_{p}} f_{s}
$$

where $d=x_{0}-x_{w}$ is the deformation of the series of the two springs $K_{c}$ and $K_{p}$ $\left(K_{\text {tip }}^{-1}=K_{c}^{-1}+K_{p}^{-1}\right)$.

In this case the evaluation of the overall stiffness of the system $K_{t o t}=\frac{\partial f_{c}}{\partial d}$ is not easy (and in general quite different from the ideal value $K_{t i p}$ ), since the force due to the stiction $f_{s}$ depends on the forces applied (by the two springs) on the mass $m$ and is therefore related to $d$. By a simple inspection of the model reported in Fig. 10 it is clear that the stiffness value experienced by the object at the contact point is

$$
K_{\text {tot }}=K_{p} \quad \text { if } \quad\left|f_{c}-f_{\tau}\right| \leq f_{s}
$$

where $f_{\tau}$ is the force provided by the control (through the actuation system).

It is clear that in order to impose a desired stiffness value at the contact a simple solution consists of

1. controlling the position of the fingertip (mass $m$ )

2. modulating the stiffness of the interface $\left(K_{p}\right)$

The first condition implies, in the simple example reported in this section (where a PD controller is assumed), a high value of the proportional term $\left(K_{c} \gg K_{p}\right)$ which leads to

$$
f_{c} \approx K_{p} d+\alpha f_{s} \text { with } \alpha=\frac{K_{p}}{K_{c}+K_{p}} \ll 1
$$

Therefore the high-gain position control loop reduces the effects of friction and the other uncertainties that the system may show, and allows to impose a precise stiffness at the contact.

\footnotetext{
${ }^{1}$ A trivial Lyapunov function for the system is $V(x, \dot{x})=\frac{1}{2} K_{c}\left(x_{0}-x\right)^{2}+\frac{1}{2} K_{p}\left(x_{w}-\right.$
} $x)^{2}+\frac{1}{2} m \dot{x}^{2}$ which leads to a $\dot{V}(x, \dot{x}) \leq 0$. 


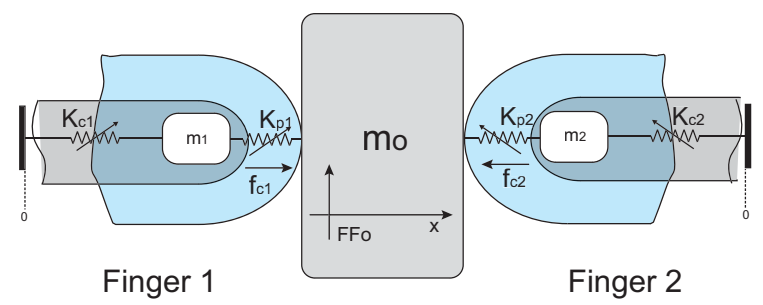

Fig. 11. Schematic model of a 2 -jaws robotic gripper grasping an object.

Stiffness control with force sensors When a sensor, able to detect the forces exerted on the environment and/or the torques provided by the actuation system to the joints of the robot finger, is available, by means of a proper control law it is possible to compensate for friction and other non-idealities which affect the system. Therefore, an ideal impedance control can be achieved. In this case, the controlled system is described by (5), where the term $f_{f}$ is neglected (obviously the same conclusions hold if the mechanical system is not affected by friction and other side effects).

The stiffness of the system is $K_{t i p}$, but, as mentioned, it can not be arbitrarily chosen by modifying $K_{c}$, since the interface is quite soft and $K_{t i p} \leq K_{p}$.

Modulating the stiffness of the interface: the role of internal forces In the example illustrated above, it is clear the need of modulating the stiffness of the interface $K_{p}$ in order to obtain the desired stiffness level for the robotic fingers, and accordingly for the hand/object. In a robotic end-effector, this can be done by adopting finger-pads that can be actively controlled [19] or (this is the case treated in this paper) by using a passive interface, whose stiffness is not constant but can be modified according to specific needs. As reported in Sec. 2.3 the stiffness of a soft pad covering an endoskeletal structure is a function of the imposed strength(/deformation). Therefore, it is possible to modify the stiffness of such a passive interface by varying the forces applied by the fingers on the object. We consider a pinch grasp by means of two 1-dof fingers, as depicted in Fig. 11. In order to change the stiffness $\left(K_{p i}\left(f_{c i}\right)\right)$ of the pad, one can vary the active internal forces ${ }^{2}$ [20] applied by the fingers:

$$
\left\{\begin{array}{l}
f_{c 1}=\lambda \\
f_{c 2}=-\lambda
\end{array}\right.
$$

with $\lambda$ positive parameter. The result is a null net force on the object.

\footnotetext{
${ }^{2}$ In the case of a position controlled end-effector, the position set-points must be varied in such a way that no motion of the object is produced.
} 


\section{Stiffness control of a "soft" hand: general approach}

\subsection{Preliminary considerations}

Given a robotic hand, grasping an object by means of $k$ fingers, the wrench $F_{o} \in \mathbb{R}^{6}$ applied to the object by the contact has the form

$$
F_{o}=G f_{c}
$$

where $f_{c}=\left[f_{c 1}^{T} f_{c 2}^{T} \ldots f_{c n_{f}}^{T}\right]^{T} \in \mathbb{R}^{m}\left(m=n_{f} \cdot s\right.$, where $s$ denotes the number of independent forces that can be applied by the contact: $s=1$ for frictionless point contact; $s=3$ for point contact with friction; $s=4$ for soft finger contact; $s=6$ for very-soft finger model) is the vector collecting the generalized contact forces $f_{c i}$ of each finger, and $G=\left[G_{1} G_{2} \ldots G_{n_{f}}\right] \in \mathbb{R}^{6 \times m}$ is the so called grasp matrix [21], which takes into account the geometric configuration of the grasp and the contact model assumed.

In the hypothesis that $F_{o}$ belongs to the range space of $G$, (7) can be solved with respect to $f_{c}$, but, since in general it has more unknowns that equations, the solution is not unique. The general solution can be written as:

$$
f_{c}=G^{R} F_{o}+N \lambda
$$

where $G^{R}$ is a right-inverse of the grasp matrix, $N \in \mathbb{R}^{m \times h}$ is a matrix whose column form a basis of the nullspace of $G$, and $\lambda \in \mathbb{R}^{h}$ is a coefficient vector which parameterizes the homogeneous parts of the solution (8). This term $(N \lambda)$ represents the internal forces of the grasp, i.e. self-balanced forces that have not effect on the net wrench $F_{o}$ on the object, but have a noticeable effect on the stability of the grasp (i.e. they are in general exploited to fulfill the constraints due to frictional contact).

By classical considerations about the duality of forces and velocities, from (7) it follows that

$$
G^{T} V_{o}=v_{c}
$$

where $V_{o} \in \mathbb{R}^{6}$ is the object speed and $v_{c} \in \mathbb{R}^{m}$ the vector of the speeds of the contact points. The same expression can be used as a first order approximation of the relation, which ties infinitesimal displacement of the fingers $\left(\delta x_{c}\right)$ with an infinitesimal object motion $\delta X_{o} \in \mathbb{R}^{6}$ :

$$
G^{T} \delta X_{o}=\delta x_{c}
$$

\subsection{Stiffness relation between object space and fingertip/joint space}

The stiffness of a grasp represents a linear approximation of the relation between the displacements imposed to the object and the resulting forces

$$
K_{o}=\frac{\partial F_{o}}{\partial X_{o}}
$$


From (10) and (7) it follows immediately that

$$
K_{o}=\underbrace{G \frac{\partial f_{c}}{\partial X_{o}}}_{K_{F}}+\underbrace{\frac{\partial G}{\partial X_{o}} f_{c}}_{K_{G}}
$$

It is widely known that the overall stiffness $K_{o}$ is the result of two "elastic" contributions, acting in parallel: the term $K_{F}$, related to the contributions of the servo-motors and of the structure, and $K_{G}$, which represents the effects of small change in the grasp configuration [15]. The main focus of this paper concerns the influence of the structural compliance (and in particular of the finger pads) on the overall hand stiffness; therefore, the contribution of $K_{G}$ (usually quite small, null if no rolling occurs) is neglected.

By applying the chain-rule to (11), one achieves

$$
K_{o} \approx K_{F}=G \frac{\partial f_{c}}{\partial x_{c}} G^{T}
$$

where

$$
\frac{\partial f_{c}}{\partial x_{c}}=K_{f}=\left[\begin{array}{cccc}
K_{11} & K_{12} & \ldots & K_{1 n_{f}} \\
K_{21} & K_{22} & \ldots & K_{2 n_{f}} \\
\vdots & \vdots & \ddots & \vdots \\
K_{n_{f} 1} & K_{n_{f} 2} & \ldots & K_{n_{f} n_{f}}
\end{array}\right]
$$

is the fingers stiffness matrix in the fingertip space $(m \times m)$. The matrix $K_{f}$ has the same structure of (13):

$$
\begin{aligned}
\left(K_{f}^{-1}\right)=\Lambda_{f}=\frac{\partial x_{c}}{\partial f_{c}} & =\frac{\partial x_{p}\left(f_{c}\right)}{\partial f_{c}}+K_{s}^{-1}+K_{c}^{-1} \\
& =\Lambda_{p}+\Lambda_{s}+\Lambda_{c}
\end{aligned}
$$

where $\Lambda_{f}, \Lambda_{p}, \Lambda_{s}, \Lambda_{c}$ are respectively the compliance of the fingers, of the pads, of the mechanical structure and of the servo mechanism (expressed in the fingertip space).

\subsection{Control of the hand stiffness}

From (12) and (13) it follows that, given the desired stiffness matrix of the object $K_{o}$, or equivalently ${ }^{3}$ the compliance $\Lambda_{o}$ and known the stiffness of the pads $K_{p}$ (while the stiffness of the structure is neglected), the matrix of the stiffness due to the control (in the fingertip space) can be computed according to

$$
K_{c}=(G^{T} \underbrace{\left(\Lambda_{o}-\left(G K_{p} G^{T}\right)^{-1}\right)}_{\Lambda_{c o}} G)^{-1}
$$

\footnotetext{
${ }^{3}$ It is assumed that $K_{o}$, which is the target of this control strategy and is therefore arbitrarily chosen, is symmetric and positive-definite and, therefore, invertible.
} 
At this point, the main issue concerns the positive-definiteness of $K_{c}$. In order to obtain a feasible control gain $K_{c}$, it is necessary to guarantee that $\Lambda_{c o}$, which represents the compliance due to the control gain, as view in the object's frame, is definite-positive. This is not easy, because $\Lambda_{c o}=\Lambda_{o}-\left(G K_{p} G^{T}\right)^{-1}$ is the result of the difference of two quantities (which are both definite-positive) but some considerations can be drawn:

- a "large" $K_{p}$ produces a "small" term $\left(G K_{p} G^{T}\right)^{-1}$ which, in the case of very stiff fingertip interfaces, can be neglected;

- $\left(G K_{p} G^{T}\right)^{-1}$ depends on the stiffness value of the pads and on the geometry of the grasp as well;

- a $\Lambda_{c o}<0$ reflects the fact that because of the large compliance of the soft pads it is impossible to obtain the desired stiffness by changing the gains of servo loops;

- by changing (an in particular by increasing) the value of the elements of $K_{p}$, it is possible to make $\Lambda_{c o}>0$.

From the above considerations it follows that, especially in the case of desired rigid grasps, the soft pads are quite problematic and can make the solution of (14) definite negative and therefore impossible (with respect to control purposes). In the proposed approach, given $\Lambda_{o}$, we act first on the contact forces (by means of the internal ones, $N \lambda$ ) of the fingers, in order to change $K_{p}$ (which accordingly to 2.3 depend on the imposed strains/deformations) in such a way that

$$
K_{p}\left(f_{c}\right) \Longrightarrow \Lambda_{c o}>0
$$

Nevertheless, in the last step of control stiffness computation

$$
K_{c}=\left(G^{T} \Lambda_{c o} G\right)^{-1}
$$

the congruence transformation $G^{T} \Lambda_{c o} G$ produces a compliance matrix which is only positive-semidefinite ${ }^{4}$.

Alternatively, in (14), instead of $G$ can be convenient to consider an grasp matrix $G^{\prime}$, augmented by means of the elements of a basis vectors of the null space of $G$, [22]

$$
G^{\prime}=\left[\begin{array}{c}
G \\
-- \\
N^{T}
\end{array}\right]
$$

In this case, it is necessary to augment the compliance matrix $\Lambda_{o}$ with arbitrary elements (which will affect the final solution), which represent the so called "internal compliances", [22]

$$
\Lambda_{o}^{\prime}=\left[\begin{array}{cc}
\Lambda_{o} & 0 \\
0 & \Lambda_{i}
\end{array}\right]>0
$$

\footnotetext{
${ }^{4}$ Accordingly, in (16) a pseudo-inverse transformation must be considered in lieu of the simple inversion.
} 
By replacing $G$ and $\Lambda_{o}$ with $G^{\prime}$ and $\Lambda_{o}^{\prime}$ in (14) and following the above procedure, it follows that the congruence transformation in (16) provides a positive-definite $K_{c}$, since $G^{\prime}$ is a full-rank square matrix ${ }^{5}$.

Finally, the control action, in the fingertip cartesian space, can be computed as

$$
f_{\text {control }}=K_{c} \delta x+N \lambda
$$

where $\delta x$ is the vector of small generalized displacements or equivalently in the joint space

$$
\tau_{\text {control }}=J^{T} K_{c} J \delta \theta_{c}+J^{T} N \lambda
$$

where $J$ is the matrix of the Jacobians ( $\left.J=\operatorname{diag}\left\{J_{1} J_{2} \ldots J_{n_{f}}\right\}\right)$ of all the fingers involved in the grasp, and $\delta \theta$ is the vector of small joint displacements.

\section{Some examples}

In order to exemplify the procedure reported in the previous section, 2D grasp configurations have been take into account (accordingly the configuration of the object is given by $\left[\begin{array}{ll}x & y\end{array}\right]^{T}$ ). Furthermore, we have considered a point contact model with friction.

\subsection{Example 1}

In the first example, reported in Fig. 12.(a), two fingers grasp a rectangle. In this case, the desired object stiffness is

$$
K_{o}=\left[\begin{array}{ccc}
28 & 0 & 0 \\
0 & 0.8 & 0 \\
0 & 0 & 0.02
\end{array}\right]
$$

but the stiffness of the pads (computed for a normal force of $5 \mathrm{~N}$ )

$$
K_{p}=\left[\begin{array}{cccc}
11.29 & 0 & 0 & 0 \\
0 & 0.39 & 0 & 0 \\
0 & 0 & 11.29 & 0 \\
0 & 0 & 0 & 0.39
\end{array}\right]
$$

leads to

$$
\Lambda_{c o}=\left[\begin{array}{ccc}
-0.0085 & 0 & 0 \\
0 & -0.0238 & 0 \\
0 & 0 & -9.5046
\end{array}\right]
$$

${ }^{5}$ In this case, the solution, expressed by (14), can be simply computed as $K_{c}=\left(G^{\prime T} \Lambda_{o}^{\prime} G^{\prime}-\right.$ $\left.K_{p}^{-1}\right)^{-1}$ 


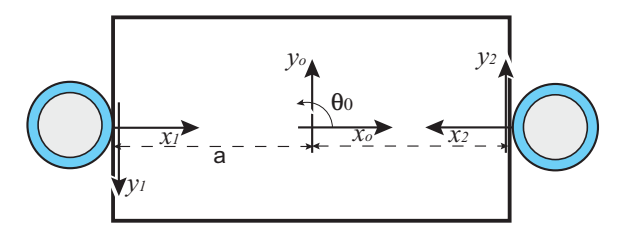

(a)

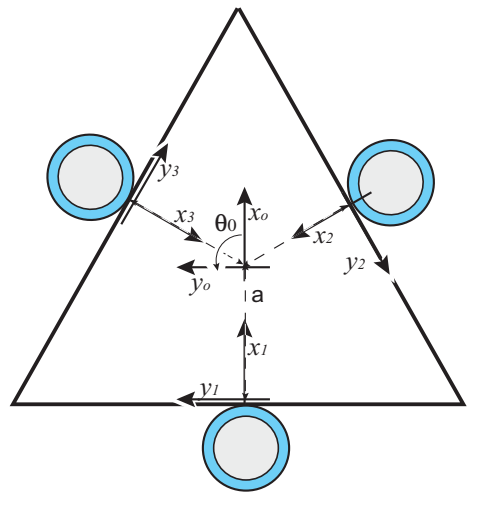

(b)

Fig. 12. Grasping of a rectangular (a) and of a triangular (b) object in $2 \mathrm{D}$ space.

which is negative-definite. By increasing the normal forces up to $9 N$, the stiffness of the soft interface becomes

$$
K_{p}=\left[\begin{array}{cccc}
16.64 & 0 & 0 & 0 \\
0 & 0.50 & 0 & 0 \\
0 & 0 & 16.64 & 0 \\
0 & 0 & 0 & 0.50
\end{array}\right]
$$

which produces

$$
\Lambda_{c o}=\left[\begin{array}{ccc}
0.0057 & 0 & 0 \\
0 & 0.2588 & 0 \\
0 & 0 & 103.51
\end{array}\right]>0
$$

Finally, the matrix of the control gains is

$$
K_{c}=\left[\begin{array}{cccc}
44.12 & 0 & -44.12 & 0 \\
0 & 1.93 & 0 & 0 \\
-44.12 & 0 & 44.12 & 0 \\
0 & 0 & 0 & 1.93
\end{array}\right] \geq 0
$$

positive-semidefinite. By considering the matrix $G^{\prime}\left(\Lambda_{i}=[1]\right)$ one achieves

$$
K_{c}^{\prime}=\left[\begin{array}{cccc}
44.65 & 0 & -43.58 & 0 \\
0 & 1.93 & 0 & 0 \\
-43.58 & 0 & 44.65 & 0 \\
0 & 0 & 0 & 1.93
\end{array}\right]>0
$$




\subsection{Example 2}

In the second example, reported in Fig. 12.(b), three fingers grasp a triangular object. The desired object stiffness is

$$
K_{o}=\left[\begin{array}{ccc}
16.95 & 0 & 0 \\
0 & 19.95 & -38.1 \\
0 & -38.1 & 79.8
\end{array}\right]
$$

and considering the stiffness of the pads, corresponding to a normal force of $5 \mathrm{~N}$ $\left(f_{c}=\lambda \cdot\left[\begin{array}{lllll}1 & 0 & 1 & 0 & 1\end{array}\right]^{T} \in \operatorname{Null}(G)\right)$

$$
\Lambda_{c o}=\left[\begin{array}{ccc}
0.002 & 0 & 0 \\
0 & 0.12 & 0.06 \\
0 & 0.06 & 0.03
\end{array}\right]
$$

which is not-definite. By considering a vector of internal forces $f_{c}=12 \cdot\left[\begin{array}{lllll}1 & 0 & 1 & 0 & 1\end{array}\right]^{T} N$, the stiffness of the soft interface increases and accordingly

$$
\Lambda_{c o}=\left[\begin{array}{ccc}
0.02 & 0 & 0 \\
0 & 0.28 & 0.13 \\
0 & 0.13 & 0.07
\end{array}\right]>0
$$

The matrix of the control gains

$$
K_{c}=\left[\begin{array}{cccccc}
3.72 & 0 & -1.86 & 3.22 & -1.86 & 3.22 \\
0 & 0.39 & 0 & 0.19 & 0 & -0.19 \\
-1.86 & 0 & 16.56 & -1.61 & -14.70 & -1.61 \\
3.22 & 0.19 & -1.61 & 2.89 & -1.61 & 2.69 \\
-1.86 & 0 & -14.70 & -1.61 & 16.56 & -1.61 \\
3.22 & -0.19 & -1.61 & 2.69 & -1.61 & 2.89
\end{array}\right] \geq 0
$$

is positive-semidefinite. In this case, by considering the matrix $G^{\prime}\left(\Lambda_{i}=\operatorname{diag}\{101010\}\right)$ one achieves

$$
K_{c}^{\prime}=\left[\begin{array}{cccccc}
0.30 & 0 & 0.2 & -0.42 & 0.20 & -0.42 \\
0 & 0.43 & 0 & 0.15 & 0 & -0.15 \\
0.20 & 0 & 15.58 & 0.21 & -15.68 & 0.21 \\
-0.42 & 0.15 & 0.21 & -0.23 & 0.21 & -0.51 \\
0.20 & 0 & -15.68 & 0.21 & 15.58 & 0.21 \\
-0.42 & -0.15 & 0.21 & -0.51 & 0.21 & -0.23
\end{array}\right]
$$

which is not definite. This is due to the further degree of freedom represented by internal compliances, but in general the research of suitable values (which lead to $K_{c}^{\prime}>0$ ) is not trivial. 


\section{Conclusions}

In this paper the normal and tangential stiffnesses of soft materials have been experimentally investigated in order to demonstrate their suitability with the development of compliant pads for robotic hands. It has been shown that both the stiffnesses strongly depends on the applied normal load.

Then, a control approach, aiming to impose a desired stiffness of the grasp, is proposed. Despite the presence of soft elements, such as the pads, this control strategy allows to obtain very rigid grasp by exploiting the non-linear relation between the compliance of the visco-elastic material (of the pads) and the imposed load.

\section{Acknowledgement}

This work has been supported by the European project GeoPlex, IST-2001-34166. Further information at http://www.geoplex.cc.

\section{References}

1. C. Melchiorri and G. Vassura. Mechanical and control features of the university of bologna hand version 2. In IEEE/RSJ International Conference on Intelligent Robots and Systems, IROS'92, Raleigh, NC, 1992.

2. J. K. Salisbury and B. Roth. Kinematics and force analysis of articulated mechanical hands. Journal of Mechanims, Transmissions and Actuation in Design, 105, 1983.

3. S.C. Jacobsen et al. Design of the utah/mit dexterous hand. In Proc. IEEE International Conference on Robotics and Automation, ICRA'86, 1986.

4. S. Schulz, C. Pylatiuk, and G. Bretthauer. A new ultralight anthropomorphic hand. In Proc. IEEE International Conference on Robotics and Automation, ICRA'02, Washington, 2002.

5. L. Biagiotti, F. Lotti, C. Melchiorri, and G. Vassura. Mechatronic design of innovative fingers for anthropomorphic robot. In Proc. IEEE International Conference on Robotics and Automation, ICRA'03, Seoul, 2003.

6. K. B. Shimoga and A. A. Goldenberg. Soft robotic fingertips - part i: A comparison of construction materials. The International Journal of Robotic Research, 15(4), 1996.

7. K. B. Shimoga and A. A. Goldenberg. Soft robotic fingertips - part ii: A comparison of construction materials. The International Journal of Robotic Research, 15(4), 1996.

8. M. R. Cutkosky and P. K. Wright. Friction, stability and the design of robotic fingers. International Journal of Robotic Research, 5(4), 1986.

9. Y. Li and I.Kao. A review of modeling of soft-contact finger and stiffness control of dexterous manipulation in robotic. In Proc. IEEE Conference on Robotic and Automation, ICRA'01, Seoul, Korea, 2001.

10. H.-Y. Han and S. Kawamura. Analysis of stiffness of human fingertip and comparison with artificial fingers. In Proc. of IEEE International Conference on Systems, Man and Cybernetics, 1999.

11. J-C Liao and M. A. Srinivasan. Experimental investigation of frictional properties of the human fingerpad. RLE Technical Report 629, M.I.T., Massachusetts, September 1999. 
12. H.-Y. Han, A. Shimada, and S. Kawamura. Analysis of friction on human fingers and design og artificial fingers. In Proc. of IEEE International Conference on Robotics and Automation, ICRA'96, Minneapolis, Minnesota, 1996.

13. N. Xydas and I. Kao. Modeling of contact mechanics and friction limit surface for soft fingers in robotics, with experimental results. The International Journal of Robotic Research, 18(8), 1999.

14. K. L. Johnson. Contact Mechanics. Cambridge University Press, 1985.

15. M. R. Cutkosky and I. Kao. Computing and controlling the compliance of a robotic hand. IEEE Transactions on Robotics and Automation, 5(2), 1989.

16. A. Bicchi, S.L. Rizzini, and G. Tonietti. Compliant design for intrinsic safety: general issues and preliminary design. In IEEE/RSJ International Conference on Intelligent Robots and Systems, IROS'01, Maui, Hawaii, 2001.

17. P. Tiezzi, F. Lotti, and G. Vassura. Polyurethane gel pulps for robotic fingers. In Proc. of IEEE International Conference on Advanced Robotics, ICAR'03, Coimbra, Portugal, 2003.

18. N. Diolaiti, C. Melchiorri, and S. Stramigioli. Contact impedance estimation for robotic systems. In submitted to IEEE/RSJ International Conference on Intelligent Robots and Systems, IROS'04, Sendai, Japan, 2004.

19. P.N. Akella and M. R. Cutkosky. Contact transition control with semiactive soft fingertips. IEEE Transactions on Robotics and Automation, 11(6), 1995.

20. A. Bicchi. On the problem of decomposing grasp and manipulation forces in multiple whole-limb manipulation. International Journal of Robotics and Autonomous Systems, vol. 13, 1994.

21. R. M. Murray, Z. Li, and S. S. Sastry. A Mathematical Introduction to Robotic Manipulation. CRC Press, Inc., Boca Raton, FL, 1994.

22. J. K. Salisbury. Kinematic and Force Analysis of Articulated Hands. $\mathrm{PhD}$ thesis, Stanford University, May 1982. 